\title{
Article \\ Evaluation of Response to Immune Checkpoint Inhibitors Using a Radiomics, Lesion-Level Approach
}

\author{
Chorog Song ${ }^{1,+}$, Hyunjin Park ${ }^{2,3,+} \oplus$, Ho Yun Lee ${ }^{1,4, *}$, Seunghak Lee ${ }^{5}$, Joong Hyun Ahn ${ }^{6}$ and Se-Hoon Lee ${ }^{4,7}$ \\ 1 Department of Radiology and Center for Imaging Science, Samsung Medical Center, \\ Sungkyunkwan University School of Medicine, Seoul 06351, Korea; chorog.song@samsung.com \\ 2 School of Electronic and Electrical Engineering, Sungkyunkwan University, Suwon 16419, Korea; \\ hyunjinp@skku.edu \\ 3 Center for Neuroscience Imaging Research, Institute for Basic Science, Suwon 16419, Korea \\ 4 Department of Health Sciences and Technology, Samsung Advanced Institute for Health Sciences \& \\ Technology (SAIHST), Sungkyunkwan University, Seoul 06355, Korea; shlee119@skku.edu \\ 5 Core Research \& Development Center, Korea University Ansan Hospital, Ansan 15355, Korea; \\ seunghaklee@korea.ac.kr \\ 6 Biostatistics and Clinical Epidemiology Center, Samsung Biomedical Research Institute, Seoul 135710, Korea; \\ jhguy.ahn@samsung.com \\ 7 Division of Hematology-Oncology, Department of Medicine, Samsung Medical Center, \\ Sungkyunkwan University School of Medicine, Seoul 06351, Korea \\ * Correspondence: hoyunlee96@gmail.com; Tel.: +82-2-3410-2502; Fax: +82-2-3410-0049 \\ + These authors contributed equally to this study.
}

check for updates

Citation: Song, C.; Park, H.; Lee, H.Y.; Lee, S.; Ahn, J.H.; Lee, S.-H. Evaluation of Response to Immune Checkpoint Inhibitors Using a Radiomics, Lesion-Level Approach. Cancers 2021, 13, 6050. https: / / doi.org/10.3390/cancers13236050

Academic Editor: David Wong

Received: 27 October 2021

Accepted: 28 November 2021

Published: 1 December 2021

Publisher's Note: MDPI stays neutral with regard to jurisdictional claims in published maps and institutional affiliations.

Copyright: (c) 2021 by the authors. Licensee MDPI, Basel, Switzerland. This article is an open access article distributed under the terms and conditions of the Creative Commons Attribution (CC BY) license (https:/ / creativecommons.org/licenses/by/ $4.0 /)$.
Simple Summary: Unique responses such as hyperprogressive disease (HPD) and a dissociated response (DR) have been reported after immune checkpoint inhibitor (ICI) treatment, and these patterns are difficult to evaluate with conventional methods. The aim of this study was to evaluate radiomics features of HPD at the lesion level, and to understand the clinical significance of a dissociated response. Our study revealed organ-specific radiomics features, likely reflecting the organ-specific microenvironment, that can be used to discriminate HPD. In addition, we observed that a dissociated response was associated with poor overall survival. A radiomic lesion-level approach shows great potential for response evaluation of ICI treatment.

Abstract: Conventional methods to determine the response to immune checkpoint inhibitors (ICIs) are limited by the unique responses to an ICI. We performed a radiomics approach for all measurable lesions to identify radiomic variables that could distinguish hyperprogressive disease (HPD) on baseline CT scans and classify a dissociated response (DR). One hundred and ninety-six patients with advanced lung cancer, treated with ICI monotherapy, who underwent at least three CT scans, were retrospectively enrolled. For all 621 measurable lesions, HPDv was determined from baseline CT scans using the tumor growth kinetics (TGK) ratio, and radiomics features were extracted. Multivariable logistic regression analysis of radiomics features was performed to discriminate DR. Radiomics features that significantly discriminated HPDv on baseline CT differed according to organ. Of the 196 patients, 54 (27.6\%) had a DR and 142 (72.4\%) did not have a DR. Overall survival in the group with a DR was significantly inferior to that in the group without a DR (log rank test, $p=0.04$ ). Our study shows that lesion-level analysis using radiomics features has great potential for discriminating HPDv and understanding heterogeneous tumor progression, including a DR, after ICI treatment.

Keywords: NSCLC; ICI; radiomics

\section{Introduction}

Lung cancer still has a poor prognosis, with the highest reported incidence and mortality among various malignancies [1]. Immune checkpoint inhibitors (ICIs) have 
recently been spotlighted for the treatment of lung cancer, as they improve outcomes, and have been rapidly adopted as a first-line treatment [2-5].

Conventional response assessment criteria such as the RECIST and WHO criteria are known to have limitations [6], and these limitations are even more pronounced when evaluating the response to ICI treatment due to the unique characteristics of ICIs [7]. Modified criteria such as the immune-related response criteria (irRC), immune-related RECIST (irRECIST), and modified RECIST 1.1 for immune-based therapeutics (termed iRECIST), have been proposed as alternatives [8-10], but these criteria only consider pseudoprogression [11].

In immunotherapy, a response pattern referred to as a dissociated response (DR) appears frequently; this pattern is characterized by the growth of some individual tumors and a decrease in size of others, which is clinically important and related to prognosis [12-14]. In addition, rapid disease progression may occur after the initiation of ICI, referred to as hyperprogressive disease (HPD), which has adverse clinical outcomes $[15,16]$.

Since atypical response patterns such as DR and HPD refer to the response patterns of individual tumors, accurate understanding, consideration, and evaluation of these phenomena is possible only by using a lesion-level approach [17]. Using radiomic analysis, quantitative information can be extracted from medical images and analyzed to obtain a comprehensive understanding of the characteristics of individual tumors [18]. Recent studies have found that radiomic features have the potential to predict treatment response [19,20] and clinical outcomes [21-24].

Our aim in this study was to use a radiomics approach to determine if measurable lesions on baseline CT images of patients with stage IV non-small cell lung cancer (NSCLC) undergoing ICI had radiomic features consistent with HPD or not. In addition, we investigated the clinical potential of using lesion-level analysis to discriminate concordant/discordant responses.

\section{Materials and Methods}

Institutional review board (IRB 2021-03-024) approval was obtained for this retrospective study with a waiver of the requirement for informed consent.

\subsection{Patients}

We retrospectively identified patients with advanced NSCLC who received ICI treatment targeting either programmed death 1 (PD-1) or its ligand (PD-L1) between 1 July 2014 and 31 July 2018 at Samsung Medical Center. Only those patients who underwent ICI monotherapy were included. The exclusion criteria for $\mathrm{CT}$ imaging review were as follows: (a) slice thickness $>2.5 \mathrm{~mm}$, (b) non-contrast CT, (c) unable to review tumor status due to superimposed acute illness, and/or (d) absence of at least three consecutive CT scans (pre-baseline, baseline or follow-up) (Figure 1). Consequently, patients treated with single immunotherapy with at least three consecutive CT scans were included in our study.

\subsection{Imaging}

CT images were obtained with the following parameters: detector collimation, 1.25 or $0.625 \mathrm{~mm} ; 120 \mathrm{kVp} ; 150-200 \mathrm{~mA}$; and reconstruction interval 1-2.5 mm. All images were displayed at standard mediastinal (window width, 400 Hounsfield units [HU]; window level, $20 \mathrm{HU}$ ) and lung (window width, $1500 \mathrm{HU}$; window level, $-700 \mathrm{HU}$ ) window settings. All CT scans were obtained after an injection of $80 \mathrm{cc}$ of contrast material at $2 \mathrm{cc} / \mathrm{s}$ followed by a normal saline flush of $20 \mathrm{cc}$ at $2 \mathrm{cc} / \mathrm{s}$.

All measurable lesions per patient were included in the analyses. A lesion-based volumetry was performed for each lesion. Lesions with volumetry values were defined as index lesions, and for each index lesion, the region of interest was segmented using commercial software (Aview, version 1.0.23, 2018; Coreline Soft, Seoul, Korea) [25,26], and then radiomic features were extracted. 
Advanced NSCLC patients treated with immunotherapy (2014.7 2018.7) $(n=472)$

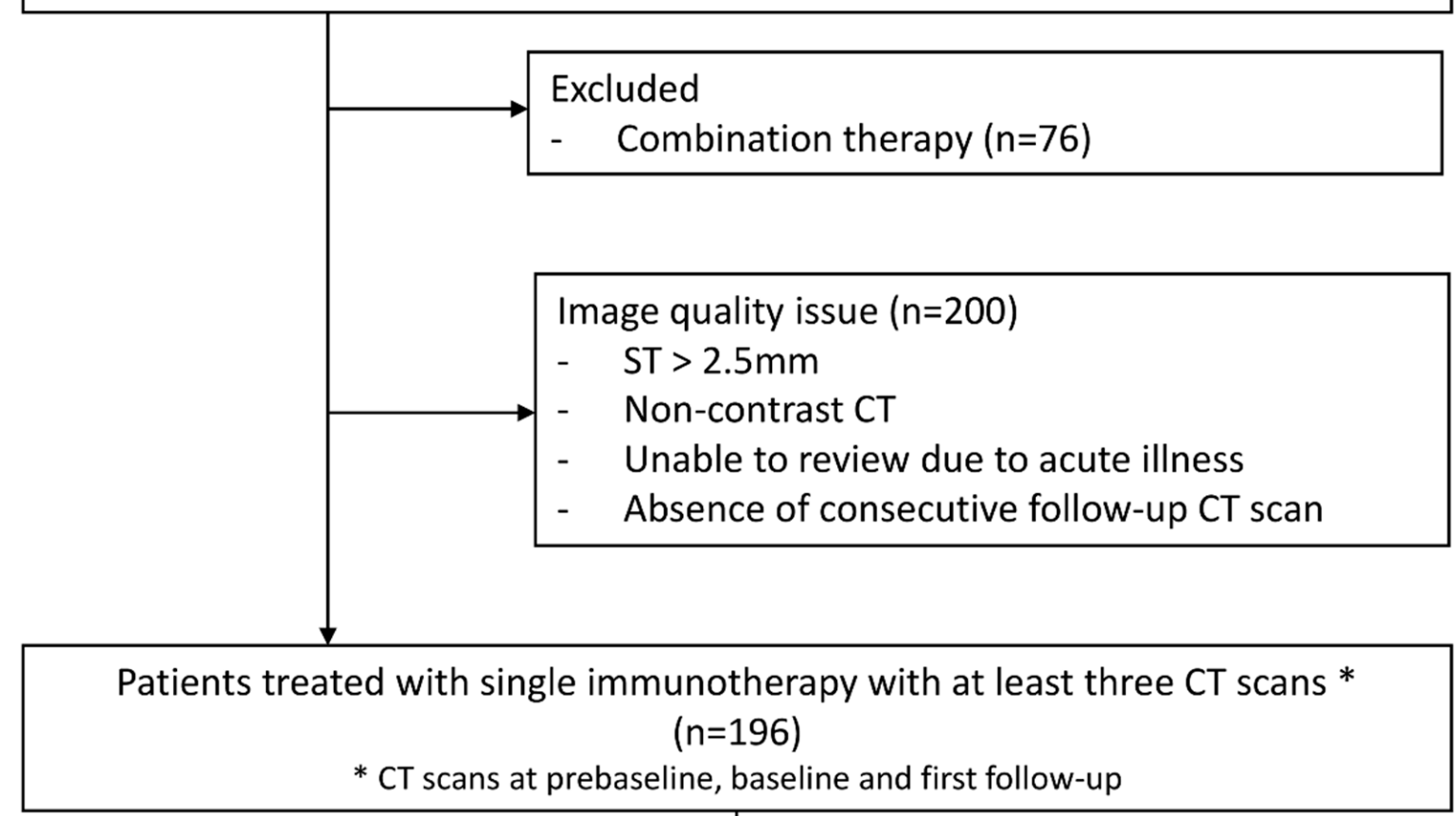

Determine HPD for each lesion

- HPD lesion $(n=147)$

- Non-HPD lesion ( $n=349)$

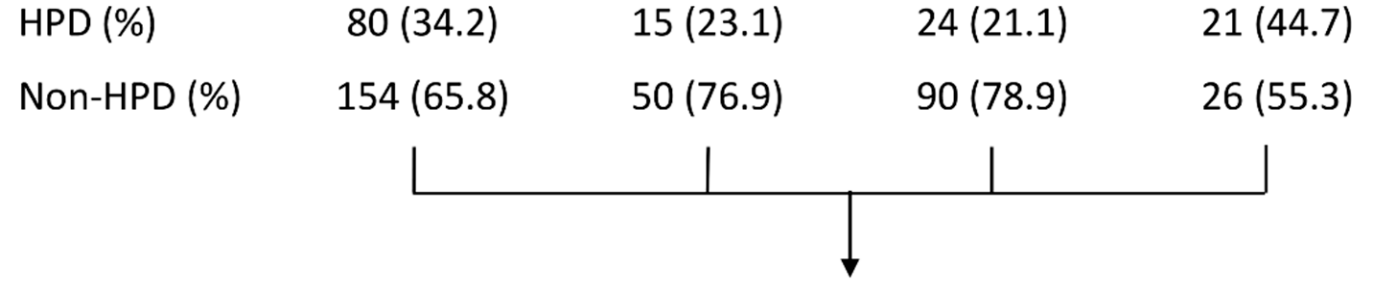

Radiomic feature extraction from baseline CT to predict HPD

Figure 1. Flowchart of patient enrollment, exclusion, and our lesion-based radiomics approach. HPD, hyperprogressive disease; LN, lymph node; NSCLC, non-small cell lung cancer; ST, slice thickness. 
The HPD of index lesions was evaluated using the tumor growth kinetics (TGK) ratio. After determining the HPD using this formula, it was defined as HPDv lesion. The TGK ratio was calculated as the differences between tumor volumes from consecutive CT scans [27]. To overcome the limitations of RECIST when defining HPD, HPDv using volumetry was used. As a limitation of the RECIST criteria, first, the tumor burden cannot be fully reflected, and, in particular, there is a limitation that non-target lesions cannot be included. In a previous study, it was confirmed that this limitation could be improved with HPDv using the volume analysis of the entire lesion. HPDv was defined as follows: (a) time to treatment failure less than 2 months, (b) TGK ratio of 2 or more, and (3) volume increase of $50 \%$ compared with baseline [28].

To categorize dissociated and non-dissociated responses, the Nelson criteria based on volumetry values from lung nodule screening were used. Progression was defined when the volume of an individual index lesion increased by $25 \%$ or more, and a partial response was defined as a decrease of $25 \%$ or less in volume. Cases where lesions in different organs in one patient differed in progression and partial response were defined as DR cases. Patients with one target lesion were assigned to the non-DR group [29,30].

\subsection{Radiomics Analysis}

Lesions were grouped according to the organ involved, namely, lung, liver, lymph nodes, and bone. Radiomics feature extraction was performed on lesions visualized in baseline CT scans. Many small lesions were present in organs and we had to model many different organs; therefore, 19 histogram-based radiomics features and area features (2.5 D) for each organ were computed using an in-house MATLAB code (Mathworks Inc., Natick, MA, USA) and Pyradiomics [31]. Further details regarding the category and number of radiomics features adopted are provided in Table S5.

\subsection{Statistical Analyses}

Prior to statistical analyses, some features were log- or power- $(1 / 2$ and $1 / 3)$ transformed to compress the distribution of the features. For data that included negative numbers, cube conversion was performed.

We analyzed the 29 significant radiomics features, including transformed features $(n=14)$ and the original features $(n=20)$, to identify factors capable of discriminating HPDv using baseline CT data. The $p$-values of all 29 radiomics features from baseline CT scans were calculated using univariate generalized estimating equations (GEEs). Features with a $p$-value $<0.1$ were selected as significant for HPDv discrimination.

Next, we analyzed the selected features using multiple GEEs. We performed subgroup analysis according to the involved organ. Furthermore, receiver operating characteristic (ROC) analysis with a calculation of the area under the receiver operating characteristic curve (AUC) was conducted to determine the sensitivity and specificity of various features with regard to their ability to discriminate HPDv.

The overall survival (OS) was calculated as the time interval between the first administration of an ICI and death due to any cause. Living patients were right-censored at the time of last contact. The Kaplan-Meier method was used to estimate the cumulative probability of overall survival of patients treated with immunotherapy. Differences between survival curves were analyzed with the log-rank test. Statistical analysis was performed using SPSS $^{\circledR}$ v17.0 for Windows (SPSS, Inc., Chicago, IL, USA) and the SAS ${ }^{\circledR}$ software package (SAS Institute, Inc., Cary, NC, USA).

\section{Results}

\subsection{Baseline Characteristics}

A total of 472 patients, treated with ICIs between July 2014 and July 2018, were screened. Patients administered a combination of ICIs were excluded $(n=76)$. Patients with image quality issues were also excluded $(n=200)$ (Figure 1$)$. 
We analyzed 196 patients treated with ICI monotherapy for whom at least three CT scans were available. The best overall responses assessed by RECIST 1.1 were partial response (PR) in 31 patients $(15.8 \%)$, stable disease (SD) in 24 patients (12.2\%), and progressive disease (PD) in 141 patients (71.9\%).

\subsection{Determination of HPDv at the Lesion Level}

We extracted the tumor volume for each lesion and examined variations in TGK for 621 lesions. Among the 621 lesions, there were 147 HPDv lesions (23.7\%) and 349 nonHPDv lesions (56.2\%). HPDv is defined as growth that is more than double that of tumor growth before the ICI treatment [28]. Therefore, in order to determine HPDv, it is necessary to assume that the lesion must be present in all three images: pre-baseline, baseline, and first follow-up. Therefore, 125 lesions that were not in the pre-baseline, or disappeared at the first follow-up, were excluded.

The numbers of HPDv lesions in the lungs, bone, lymph nodes, and liver were $80(54.4 \%), 15(10.2 \%), 24(16.3 \%)$, and $21(14.3 \%)$, respectively. The number of non-HPDv lesions in the lungs, bone, lymph nodes, and liver were $154(44.1 \%), 50$ (14.3\%), 90 (25.8\%), and $26(7.4 \%)$, respectively. There was no significant difference in clinicopathologic variables between HPDv and non-HPDv lesions (Tables S1 and S2).

\subsection{Univariate and Multivariate Analyses}

Univariate analyses of 29 variables identified 15 variables that were significantly associated with HPDv. In the subgroup analysis by organ, there were nine variables for the lungs, four variables for the bone, nine variables for the lymph nodes, and one for the liver. These statistically significant variables are listed in Table S3.

Table 1 shows the results of the multivariate analysis of radiomic features. In the lung, the significant radiomic variables were Log (Uniformity_HIST $\left.{ }^{*} 1000\right)(p=0.001, \mathrm{OR}=0.29)$ and $\log ($ Volume $)(p=0.002, \mathrm{OR}=0.71)$, and the area under the receiver operating characteristic curve (AUC) value of these two was 0.65 ( $p$ value $<0.002)$. In the bone, Log (Uniformity_HIST * 1000) $(p=0.017, \mathrm{OR}=4.49)$ was a significant variable with an AUC of 0.70. In the lymph nodes and liver, $\log (\mathrm{RMS})(p=0.025, \mathrm{OR}=3.88)$ with an AUC of 0.63 and Percentile histogram 2.5 (Cube-root transformation) $(p=0.006, \mathrm{OR}=0.74$ ) with an AUC of 0.72 were significant variables, respectively.

Table 1. Multivariate analysis of radiomics data according to organ.

\begin{tabular}{|c|c|c|c|c|}
\hline Organ & Variable & $p$-Value & OR & $\operatorname{AUC}(95 \% \mathrm{CI})$ \\
\hline \multirow{2}{*}{ All } & \multirow{2}{*}{$\begin{array}{l}\text { Kurtosis_HIST } \\
\text { Percentile histogram } 2.5 \\
\text { (Cube-root transformation) }\end{array}$} & 0.030 & $0.98(0.96,0.99)$ & \multirow{2}{*}{$0.61(0.55-0.66)$} \\
\hline & & 0.012 & $0.94(0.89,0.98)$ & \\
\hline \multirow{2}{*}{ Lung } & Log(Uniformity_HIST*1000) & 0.001 & $0.29(0.13,0.61)$ & \multirow[t]{2}{*}{$0.65(0.58-0.72)$} \\
\hline & $\log ($ Volume $)$ & 0.002 & $0.71(0.58,0.88)$ & \\
\hline Bone & Log(Uniformity_HIST*1000) & 0.017 & $4.49(1.31,15.37)$ & $0.70(0.56-0.85)$ \\
\hline Lymph nodes & $\log (\mathrm{RM} S)$ & 0.025 & $3.88(1.18,12.70)$ & $0.63(0.49-0.77)$ \\
\hline Liver & $\begin{array}{l}\text { Percentile histogram } 2.5 \\
\text { (Cube-root transformation) }\end{array}$ & 0.006 & $0.74(0.60,0.91)$ & $0.72(0.57-0.88)$ \\
\hline Others & Median_HIST & 0.025 & $1.05(1.00,1.09)$ & $0.70(0.48-0.92)$ \\
\hline
\end{tabular}

\subsection{Prognosis Analysis}

Of the 196 patients, 54 (27.6\%) had a DR and 142 (72.4\%) did not have a DR (Table S4). OS in the group with a DR was significantly inferior to that in the group without a DR (log rank test, $p=0.04$ ) (Figure 2 ).

In the analysis according to response group, there was a significant difference in the overall survival, especially in the PR and SD groups (Figure S1). 


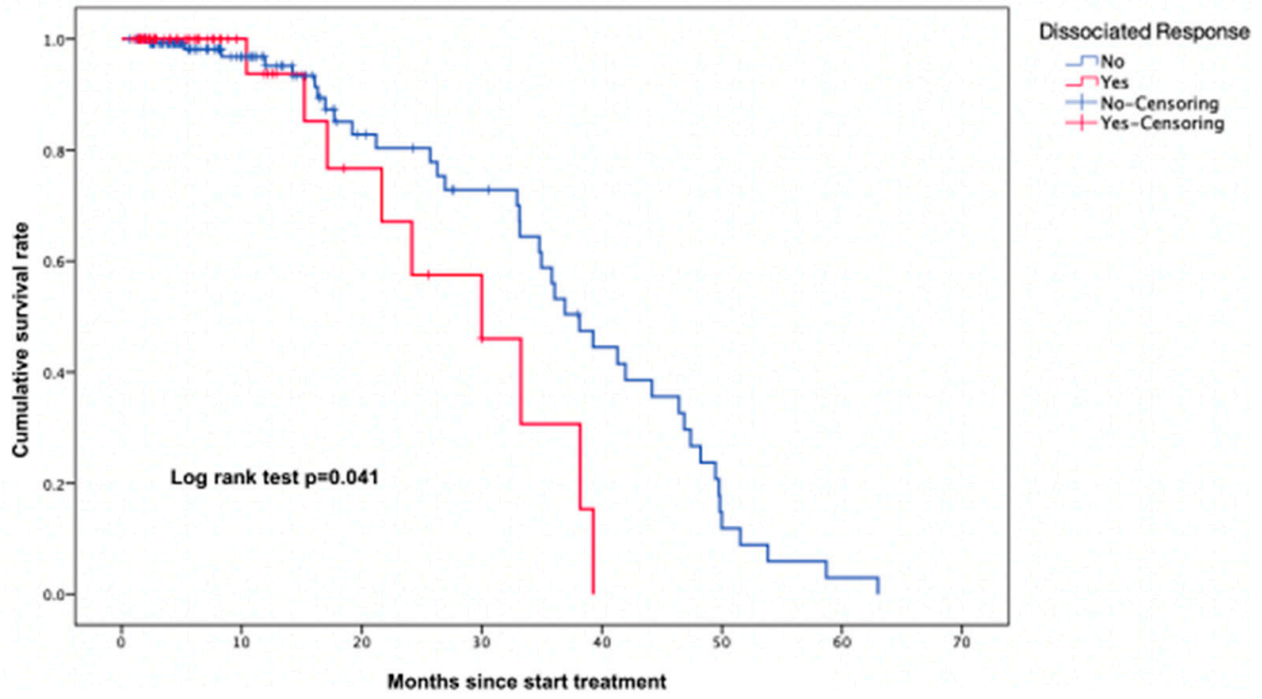

No. at Risk

$\begin{array}{rrrrrrrrr}\text { Non DR } & 142 & 67 & 35 & 27 & 15 & 5 & 1 & 0 \\ \text { DR } & 54 & 15 & 8 & 5 & 0 & 0 & 0 & 0\end{array}$

Figure 2. Comparison of overall survival between patients with a DR and those without a DR.

\section{Discussion}

To evaluate the treatment response to ICIs, we used a lesion-level radiomics approach. We found heterogeneous ICI responses for each organ based on lesion-level radiomic features extracted from baseline CT scans. The characteristic radiomics features of lesions that were classified as HPDv differed according to organ location. Concordant and discordant responses to ICIs were classified by integrating the response evaluation for each lesion and patients with a DR showed poor outcomes.

In recent studies, heterogeneous responses from organs to ICI have been observed and metastatic liver lesions have been reported to have a particularly poor response [17,32]. Similarly, in one study, patients with liver lesions had a low overall survival rate [20]. These heterogeneous responses to ICI can be partially explained by differences in the organ-specific tumor microenvironment $[33,34]$, as the mechanism of ICI therapy is based on the effect of ICI on tumor infiltrating lymphocytes (TILs), and other immune cells in the tumor microenvironment [35].

When evaluating treatment response, it would be helpful to use a lesion-level radiomics approach to assess the unique behavior of ICI treatment. We used quantitative data extracted from baseline $\mathrm{CT}$ images for HPDv discrimination. Different radiomics variables were selected for different organs, likely reflecting the unique characteristics of each organ. Curiously, in both the lung and bone, Log(Uniformity_HIST*1000) was selected with an opposite OR direction (0.29 in lung, 4.49 in bone). Uniformity indicates the homogeneity of voxels, and we found a correlation between heterogeneous lung lesions and HPD progression. This result is consistent with the association between high tumor heterogeneity and the poor outcomes reported in previous studies [21,36]. Conversely, in the bone, the uniformity_HIST value of the metastatic lesion was 0.054 , whereas that of the reference normal vertebra was 0.035 , indicating that the metastatic lesion was rather homogenous (Figure 3). Tumor cells disrupt the normal bone remodeling process, causing the disappearance of the normal bone matrix $[37,38]$. CT images show mainly radiolucent and osteolytic lesions and are thought to be more homogenous than normal bone tissue [39]. Meanwhile, in the lymph nodes, Log (RMS) was selected, which reflects the magnitude of the histogram. A recent study by Coroller et al. showed that radiomic features indicating a sphericity of the primary tumor and lymph node homogeneity were correlated with better pathologic responses in NSCLC patients with concurrent chemoradiation therapy [40], 
which is consistent with our findings. In the case of the liver, Percentile histogram 2.5 (Cube-root transformation) was a significant variable. This variable measures the intensity values at the 2.5th percentile on the histogram and indicates that lesions with lower CT attenuation showed hyperprogression in the liver. Hepatic metastasis is frequently accompanied by necrosis, which is caused by tumor hypoxia and mainly occurs in centers with poor blood and oxygen supply [41,42]. In addition, the genomic stability of tumor cells in the tumor center is much lower, with a higher possibility of distant metastasis [43]. It is thought that necrosis of the tumor center further promotes tumor cell mutation, and that this is related to HPD.

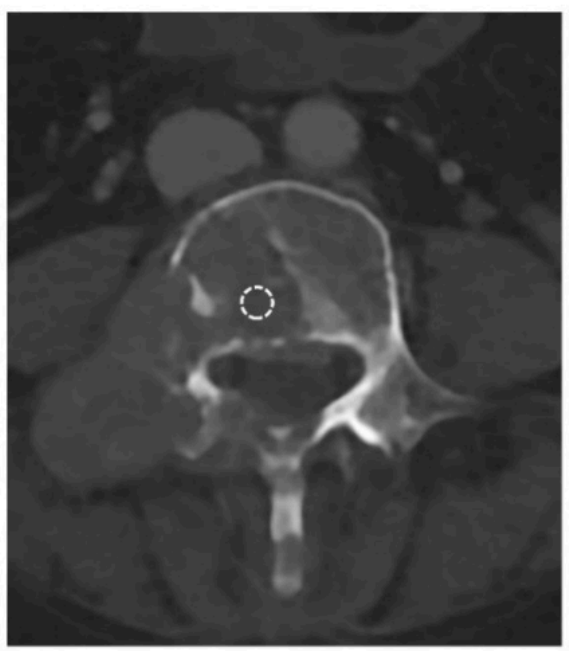

(a)

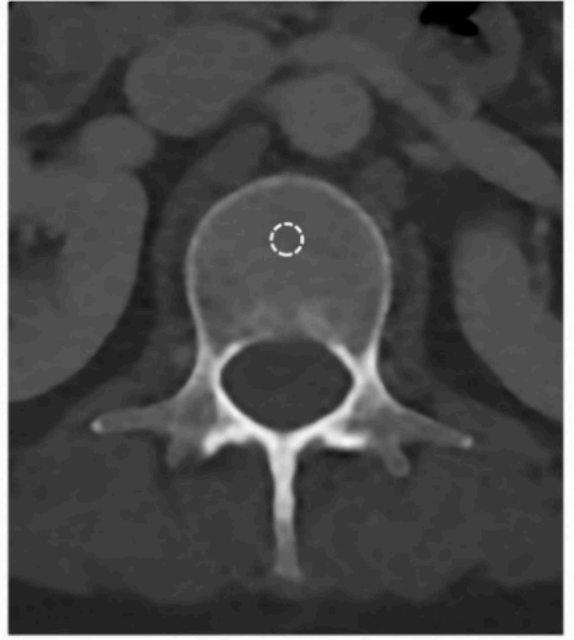

(b)

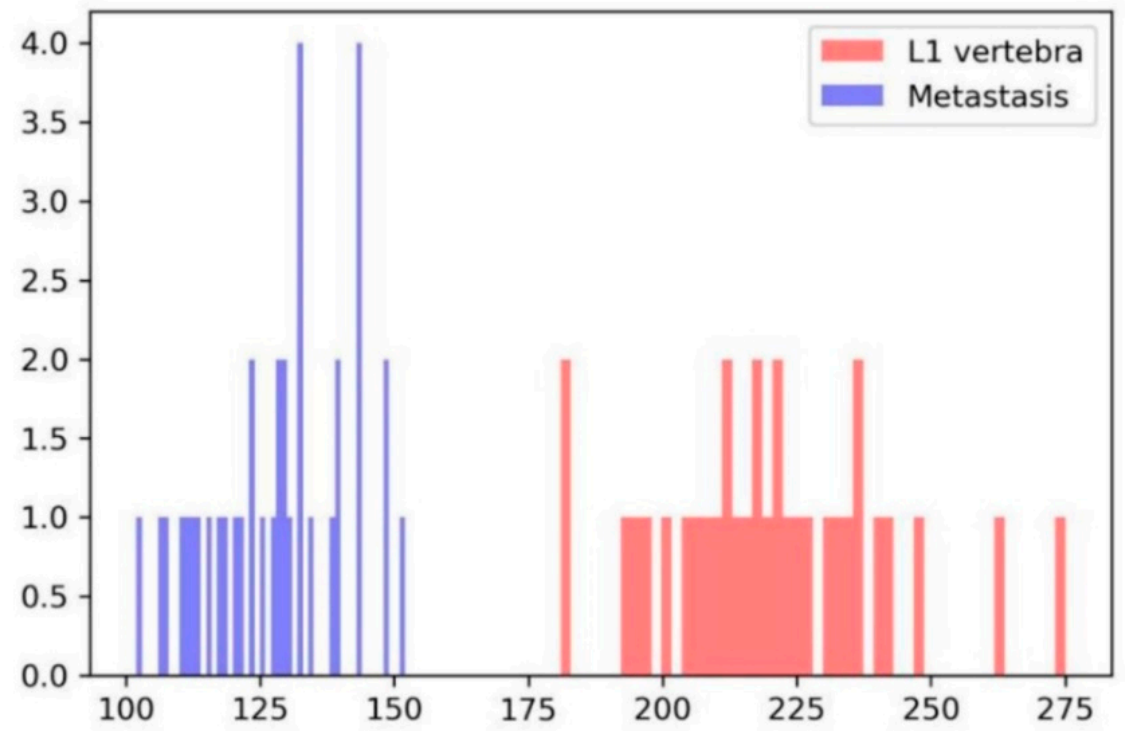

(c)

Figure 3. Baseline CT of a 47-year-old female with NSCLC showing (a) metastasis and (b) the normal L1 vertebra as a reference. (c) The uniformity of metastasis was higher than that of the normal reference, meaning that metastasis-related voxels were homogenous.

The clinical impact of a DR is unclear. In one previous study, a DR was shown to be to be associated with an unfavorable prognosis [14]. However, recent studies have reported better overall survival than true progression in patients with a DR $[12,44]$. In our study, patients with a DR had poor overall survival (Figure 2). When response groups were compared, significant differences were seen in the PR and SD groups but not the 
progression group (Figure S1). DR is associated with a poor prognosis in response group patients; therefore, it can be used as an important factor in determining whether to maintain treatment in this group. Thus, further research on response criteria, including a DR, is needed.

In addition, the process of obtaining volume data using volumetry does not require additional advanced imaging techniques. CT images already taken for treatment in daily practice are used without additional imaging or the application of other imaging techniques. If only a semiautomated tumor segmentation is performed, then the volume can be obtained by calculation using computer software, which is possible through a commercialized program or even an open-source program [45-48]. Therefore, lesion-level evaluation through volumetry is expected to have great clinical potential as well as not being difficult to apply to actual daily practice.

The limitations of our study include its single-center nature, the small sample size, and the sample from a single site. To overcome this weakness, additional external validation is needed in future multi-center large cohort studies. Next, using a lesion-level approach, it was impossible to assess for both new and disappeared lesions on follow-up which may have resulted in selection bias. However, we used an ROI segmentation and radiomics approach for all lesions present on the CT scans collected at three points in time.

\section{Conclusions}

Organ-specific radiomics features from baseline CT scans were useful for HPDv discrimination. A dissociated response was classified by integrating the findings of the response evaluation for each lesion, and a DR was shown to be correlated with a poor outcome. As a preliminary study on the usefulness of a lesion-level radiomics approach, our findings highlight the potential for using this type of approach to capture intra- and inter-tumor heterogeneity.

Supplementary Materials: The following are available online at https:/ /www.mdpi.com/article/10 .3390 / cancers13236050/s1, Figure S1: Comparison of overall survival between patients with a DR and patients without a DR. Subgroup analysis was performed by best overall response (as assessed by RECIST 1.1), Table S1: Baseline characteristics of study patients and CT, Table S2: Radiomics features of non-HPDv and HPDv lesions, Table S3: Univariate analysis of radiomics features to discriminate HPDv, Table S4: Determination of dissociated response and classification according to best response group, Table S5: Definition of extracted radiomics features.

Author Contributions: Conceptualization, H.Y.L.; Methodology, S.L.; Software, S.L.; Formal Analysis, J.H.A.; Investigation, H.Y.L.; Resources, H.Y.L.; Data Curation, C.S. and S.-H.L.; Writing-Original Draft Preparation, C.S. Writing—Review and Editing, C.S., H.Y.L., H.P. and S.L.; Visualization, C.S.; Supervision, H.Y.L.; Project Administration, H.Y.L.; Funding Acquisition, H.Y.L. All authors have read and agreed to the published version of the manuscript.

Funding: This study was supported by the Future Medicine $20 * 30$ Project of Samsung Medical Center [\#SMX1210781].

Institutional Review Board Statement: Institutional review board (IRB 2021-03-024) approval was obtained for this retrospective study with waiver of informed consent.

Informed Consent Statement: Patient consent was waived due to the minimal risk posed to patients.

Data Availability Statement: The data presented in this study are available in this article and Supplementary Materials.

Conflicts of Interest: The authors declare no conflict of interest. 


$\begin{array}{ll}\text { Abbreviations } \\ \text { AUC } & \begin{array}{l}\text { area under the receiver operating characteristic curve } \\ \text { dissociated response }\end{array} \\ \text { DR } & \text { generalized estimating equation } \\ \text { GEE } & \text { hyperprogressive disease } \\ \text { HPD } & \text { immune checkpoint inhibitor } \\ \text { ICI } & \text { modified RECIST 1.1 for immune-based therapeutics } \\ \text { iRECIST } & \text { immune-related response criteria } \\ \text { irRC } & \text { immune-related RECIST } \\ \text { irRECIST } & \text { non-small cell lung cancer } \\ \text { NSCLC } & \text { overall survival } \\ \text { OS } & \text { progressive disease } \\ \text { PD } & \text { receptor programmed cell death 1 } \\ \text { PD-1 } & \text { programmed death ligand 1 } \\ \text { PD-L1 } & \text { partial response } \\ \text { PR } & \text { receiver operating characteristic } \\ \text { ROC } & \text { stable disease } \\ \text { SD } & \text { tumor growth kinetics } \\ \text { TGK } & \end{array}$

\section{References}

1. Sung, H.; Ferlay, J.; Siegel, R.L.; Laversanne, M.; Soerjomataram, I.; Jemal, A.; Bray, F. Global Cancer Statistics 2020: GLOBOCAN Estimates of Incidence and Mortality Worldwide for 36 Cancers in 185 Countries. CA Cancer J. Clin. 2021, 71, 209-249. [CrossRef]

2. Pai-Scherf, L.; Blumenthal, G.M.; Li, H.; Subramaniam, S.; Mishra-Kalyani, P.S.; He, K.; Zhao, H.; Yu, J.; Paciga, M.; Goldberg, K.B.; et al. FDA Approval Summary: Pembrolizumab for Treatment of Metastatic Non-Small Cell Lung Cancer: First-Line Therapy and Beyond. Oncologist 2017, 22, 1392-1399. [CrossRef]

3. Antonia, S.J.; Villegas, A.; Daniel, D.; Vicente, D.; Murakami, S.; Hui, R.; Kurata, T.; Chiappori, A.; Lee, K.H.; de Wit, M.; et al. Overall Survival with Durvalumab after Chemoradiotherapy in Stage III NSCLC. N. Engl. J. Med. 2018, 379, 2342-2350. [CrossRef]

4. Hirsch, F.R.; Scagliotti, G.V.; Mulshine, J.L.; Kwon, R.; Curran, W.J., Jr.; Wu, Y.L.; Paz-Ares, L. Lung cancer: Current therapies and new targeted treatments. Lancet 2017, 389, 299-311. [CrossRef]

5. Onoi, K.; Chihara, Y.; Uchino, J.; Shimamoto, T.; Morimoto, Y.; Iwasaku, M.; Kaneko, Y.; Yamada, T.; Takayama, K. Immune Checkpoint Inhibitors for Lung Cancer Treatment: A Review. J. Clin. Med. 2020, 9, 1362. [CrossRef] [PubMed]

6. Nishino, M.; Jagannathan, J.P.; Krajewski, K.M.; O’Regan, K.; Hatabu, H.; Shapiro, G.; Ramaiya, N.H. Personalized tumor response assessment in the era of molecular medicine: Cancer-specific and therapy-specific response criteria to complement pitfalls of RECIST. AJR Am. J. Roentgenol. 2012, 198, 737-745. [CrossRef] [PubMed]

7. Chiou, V.L.; Burotto, M. Pseudoprogression and Immune-Related Response in Solid Tumors. J. Clin. Oncol. 2015, 33, 3541-3543. [CrossRef]

8. Wolchok, J.D.; Hoos, A.; O’Day, S.; Weber, J.S.; Hamid, O.; Lebbe, C.; Maio, M.; Binder, M.; Bohnsack, O.; Nichol, G.; et al. Guidelines for the evaluation of immune therapy activity in solid tumors: Immune-related response criteria. Clin. Cancer Res. 2009, 15, 7412-7420. [CrossRef]

9. Nishino, M.; Tirumani, S.H.; Ramaiya, N.H.; Hodi, F.S. Cancer immunotherapy and immune-related response assessment: The role of radiologists in the new arena of cancer treatment. Eur. J. Radiol. 2015, 84, 1259-1268. [CrossRef]

10. Seymour, L.; Bogaerts, J.; Perrone, A.; Ford, R.; Schwartz, L.H.; Mandrekar, S.; Lin, N.U.; Litiere, S.; Dancey, J.; Chen, A.; et al. iRECIST: Guidelines for response criteria for use in trials testing immunotherapeutics. Lancet Oncol. 2017, 18, e143-e152. [CrossRef]

11. Park, H.J.; Kim, K.W.; Pyo, J.; Suh, C.H.; Yoon, S.; Hatabu, H.; Nishino, M. Incidence of Pseudoprogression during Immune Checkpoint Inhibitor Therapy for Solid Tumors: A Systematic Review and Meta-Analysis. Radiology 2020, 297, 87-96. [CrossRef]

12. Tazdait, M.; Mezquita, L.; Lahmar, J.; Ferrara, R.; Bidault, F.; Ammari, S.; Balleyguier, C.; Planchard, D.; Gazzah, A.; Soria, J.C.; et al. Patterns of responses in metastatic NSCLC during PD-1 or PDL-1 inhibitor therapy: Comparison of RECIST 1.1, irRECIST and iRECIST criteria. Eur. J. Cancer 2018, 88, 38-47. [CrossRef]

13. Borcoman, E.; Kanjanapan, Y.; Champiat, S.; Kato, S.; Servois, V.; Kurzrock, R.; Goel, S.; Bedard, P.; Le Tourneau, C. Novel patterns of response under immunotherapy. Ann. Oncol. 2019, 30, 385-396. [CrossRef] [PubMed]

14. Dong, Z.Y.; Zhai, H.R.; Hou, Q.Y.; Su, J.; Liu, S.Y.; Yan, H.H.; Li, Y.S.; Chen, Z.Y.; Zhong, W.Z.; Wu, Y.L. Mixed Responses to Systemic Therapy Revealed Potential Genetic Heterogeneity and Poor Survival in Patients with Non-Small Cell Lung Cancer. Oncologist 2017, 22, 61-69. [CrossRef]

15. Ferrara, R.; Mezquita, L.; Texier, M.; Lahmar, J.; Audigier-Valette, C.; Tessonnier, L.; Mazieres, J.; Zalcman, G.; Brosseau, S.; Le Moulec, S.; et al. Hyperprogressive Disease in Patients With Advanced Non-Small Cell Lung Cancer Treated With PD-1/PD-L1 Inhibitors or With Single-Agent Chemotherapy. JAMA Oncol. 2018, 4, 1543-1552. [CrossRef] 
16. Champiat, S.; Dercle, L.; Ammari, S.; Massard, C.; Hollebecque, A.; Postel-Vinay, S.; Chaput, N.; Eggermont, A.; Marabelle, A.; Soria, J.C.; et al. Hyperprogressive Disease Is a New Pattern of Progression in Cancer Patients Treated by Anti-PD-1/PD-L1. Clin . Cancer Res. 2017, 23, 1920-1928. [CrossRef] [PubMed]

17. Osorio, J.C.; Arbour, K.C.; Le, D.T.; Durham, J.N.; Plodkowski, A.J.; Halpenny, D.F.; Ginsberg, M.S.; Sawan, P.; Crompton, J.G.; $\mathrm{Yu}$, H.A.; et al. Lesion-Level Response Dynamics to Programmed Cell Death Protein (PD-1) Blockade. J. Clin. Oncol. 2019, 37, 3546-3555. [CrossRef]

18. Chetan, M.R.; Gleeson, F.V. Radiomics in predicting treatment response in non-small-cell lung cancer: Current status, challenges and future perspectives. Eur. Radiol. 2021, 31, 1049-1058. [CrossRef]

19. Cha, K.H.; Hadjiiski, L.; Chan, H.P.; Weizer, A.Z.; Alva, A.; Cohan, R.H.; Caoili, E.M.; Paramagul, C.; Samala, R.K. Bladder Cancer Treatment Response Assessment in CT using Radiomics with Deep-Learning. Sci. Rep. 2017, 7, 8738. [CrossRef] [PubMed]

20. Sun, R.; Limkin, E.J.; Vakalopoulou, M.; Dercle, L.; Champiat, S.; Han, S.R.; Verlingue, L.; Brandao, D.; Lancia, A.; Ammari, S.; et al. A radiomics approach to assess tumour-infiltrating CD8 cells and response to anti-PD-1 or anti-PD-L1 immunotherapy: An imaging biomarker, retrospective multicohort study. Lancet Oncol. 2018, 19, 1180-1191. [CrossRef]

21. Ganeshan, B.; Panayiotou, E.; Burnand, K.; Dizdarevic, S.; Miles, K. Tumour heterogeneity in non-small cell lung carcinoma assessed by CT texture analysis: A potential marker of survival. Eur. Radiol. 2012, 22, 796-802. [CrossRef]

22. Haider, M.A.; Vosough, A.; Khalvati, F.; Kiss, A.; Ganeshan, B.; Bjarnason, G.A. CT texture analysis: A potential tool for prediction of survival in patients with metastatic clear cell carcinoma treated with sunitinib. Cancer Imaging 2017, 17, 4. [CrossRef]

23. Aerts, H.J.; Velazquez, E.R.; Leijenaar, R.T.; Parmar, C.; Grossmann, P.; Carvalho, S.; Bussink, J.; Monshouwer, R.; Haibe-Kains, B.; Rietveld, D.; et al. Decoding tumour phenotype by noninvasive imaging using a quantitative radiomics approach. Nat. Commun. 2014, 5, 4006. [CrossRef]

24. Parmar, C.; Leijenaar, R.T.; Grossmann, P.; Rios Velazquez, E.; Bussink, J.; Rietveld, D.; Rietbergen, M.M.; Haibe-Kains, B.; Lambin, P.; Aerts, H.J. Radiomic feature clusters and prognostic signatures specific for Lung and Head \& Neck cancer. Sci. Rep. 2015, 5, 11044. [CrossRef] [PubMed]

25. Choe, J.; Lee, S.M.; Do, K.H.; Lee, G.; Lee, J.G.; Lee, S.M.; Seo, J.B. Deep Learning-based Image Conversion of CT Reconstruction Kernels Improves Radiomics Reproducibility for Pulmonary Nodules or Masses. Radiology 2019, 292, 365-373. [CrossRef] [PubMed]

26. Parmar, C.; Rios Velazquez, E.; Leijenaar, R.; Jermoumi, M.; Carvalho, S.; Mak, R.H.; Mitra, S.; Shankar, B.U.; Kikinis, R.; Haibe-Kains, B.; et al. Robust Radiomics feature quantification using semiautomatic volumetric segmentation. PLoS ONE 2014, 9, e102107. [CrossRef]

27. Le Tourneau, C.; Servois, V.; Dieras, V.; Ollivier, L.; Tresca, P.; Paoletti, X. Tumour growth kinetics assessment: Added value to RECIST in cancer patients treated with molecularly targeted agents. Br. J. Cancer 2012, 106, 854-857. [CrossRef]

28. Kim, Y.; Kim, C.H.; Lee, H.Y.; Lee, S.H.; Kim, H.S.; Lee, S.; Cha, H.; Hong, S.; Kim, K.; Seo, S.W.; et al. Comprehensive Clinical and Genetic Characterization of Hyperprogression Based on Volumetry in Advanced Non-Small Cell Lung Cancer Treated With Immune Checkpoint Inhibitor. J. Thorac. Oncol. 2019, 14, 1608-1618. [CrossRef]

29. Field, J.K.; Oudkerk, M.; Pedersen, J.H.; Duffy, S.W. Prospects for population screening and diagnosis of lung cancer. Lancet 2013, 382, 732-741. [CrossRef]

30. van Klaveren, R.J.; Oudkerk, M.; Prokop, M.; Scholten, E.T.; Nackaerts, K.; Vernhout, R.; van Iersel, C.A.; van den Bergh, K.A.; van Westeinde, S.; van der Aalst, C.; et al. Management of lung nodules detected by volume CT scanning. N. Engl. J. Med. 2009, 361, 2221-2229. [CrossRef] [PubMed]

31. van Griethuysen, J.J.M.; Fedorov, A.; Parmar, C.; Hosny, A.; Aucoin, N.; Narayan, V.; Beets-Tan, R.G.H.; Fillion-Robin, J.C.; Pieper, S.; Aerts, H. Computational Radiomics System to Decode the Radiographic Phenotype. Cancer Res. 2017, 77, e104-e107. [CrossRef]

32. Tumeh, P.C.; Hellmann, M.D.; Hamid, O.; Tsai, K.K.; Loo, K.L.; Gubens, M.A.; Rosenblum, M.; Harview, C.L.; Taube, J.M.; Handley, N.; et al. Liver Metastasis and Treatment Outcome with Anti-PD-1 Monoclonal Antibody in Patients with Melanoma and NSCLC. Cancer Immunol. Res. 2017, 5, 417-424. [CrossRef]

33. Jimenez-Sanchez, A.; Memon, D.; Pourpe, S.; Veeraraghavan, H.; Li, Y.; Vargas, H.A.; Gill, M.B.; Park, K.J.; Zivanovic, O.; Konner, J.; et al. Heterogeneous Tumor-Immune Microenvironments among Differentially Growing Metastases in an Ovarian Cancer Patient. Cell 2017, 170, 927-938.e20. [CrossRef]

34. Sharma, P.; Allison, J.P. The future of immune checkpoint therapy. Science 2015, 348, 56-61. [CrossRef]

35. Cogdill, A.P.; Andrews, M.C.; Wargo, J.A. Hallmarks of response to immune checkpoint blockade. Br. J. Cancer 2017, 117, 1-7. [CrossRef]

36. Ng, F.; Kozarski, R.; Ganeshan, B.; Goh, V. Assessment of tumor heterogeneity by CT texture analysis: Can the largest crosssectional area be used as an alternative to whole tumor analysis? Eur. J. Radiol. 2013, 82, 342-348. [CrossRef]

37. Clezardin, P. Pathophysiology of bone metastases from solid malignancies. Jt. Bone Spine 2017, 84, 677-684. [CrossRef]

38. Mundy, G.R. Metastasis to bone: Causes, consequences and therapeutic opportunities. Nat. Rev. Cancer 2002, 2, 584-593. [CrossRef] [PubMed]

39. Coleman, R.E. Metastatic bone disease: Clinical features, pathophysiology and treatment strategies. Cancer Treat. Rev. 2001, 27, 165-176. [CrossRef] [PubMed] 
40. Coroller, T.P.; Agrawal, V.; Huynh, E.; Narayan, V.; Lee, S.W.; Mak, R.H.; Aerts, H. Radiomic-Based Pathological Response Prediction from Primary Tumors and Lymph Nodes in NSCLC. J. Thorac. Oncol. 2017, 12, 467-476. [CrossRef] [PubMed]

41. Sica, G.T.; Ji, H.; Ros, P.R. CT and MR imaging of hepatic metastases. AJR Am. J. Roentgenol. 2000, 174, 691-698. [CrossRef]

42. Paulatto, L.; Dioguardi Burgio, M.; Sartoris, R.; Beaufrere, A.; Cauchy, F.; Paradis, V.; Vilgrain, V.; Ronot, M. Colorectal liver metastases: Radiopathological correlation. Insights Imaging 2020, 11, 99. [CrossRef]

43. Zhao, Y.; Fu, X.; Lopez, J.I.; Rowan, A.; Au, L.; Fendler, A.; Hazell, S.; Xu, H.; Horswell, S.; Shepherd, S.T.C.; et al. Selection of metastasis competent subclones in the tumour interior. Nat. Ecol. Evol. 2021, 5, 1033-1045. [CrossRef]

44. Tozuka, T.; Kitazono, S.; Sakamoto, H.; Yoshida, H.; Amino, Y.; Uematsu, S.; Yoshizawa, T.; Hasegawa, T.; Uchibori, K.; Yanagitani, N.; et al. Dissociated responses at initial computed tomography evaluation is a good prognostic factor in non-small cell lung cancer patients treated with anti-programmed cell death-1/ligand 1 inhibitors. BMC Cancer 2020, 20, 207. [CrossRef] [PubMed]

45. Maitland, M.L. Volumes to learn: Advancing therapeutics with innovative computed tomography image data analysis. Clin Cancer Res. 2010, 16, 4493-4495. [CrossRef] [PubMed]

46. Mehta, V.A.; Sampson, J.H. The limitations of imaging response criteria. Lancet Oncol. 2012, 13, 1064-1065. [CrossRef]

47. Kang, H.; Lee, H.Y.; Lee, K.S.; Kim, J.H. Imaging-based tumor treatment response evaluation: Review of conventional, new, and emerging concepts. Korean J. Radiol. 2012, 13, 371-390. [CrossRef] [PubMed]

48. Kanaly, C.W.; Ding, D.; Mehta, A.I.; Waller, A.F.; Crocker, I.; Desjardins, A.; Reardon, D.A.; Friedman, A.H.; Bigner, D.D.; Sampson, J.H. A novel method for volumetric MRI response assessment of enhancing brain tumors. PLoS ONE 2011, 6, e16031. [CrossRef] 